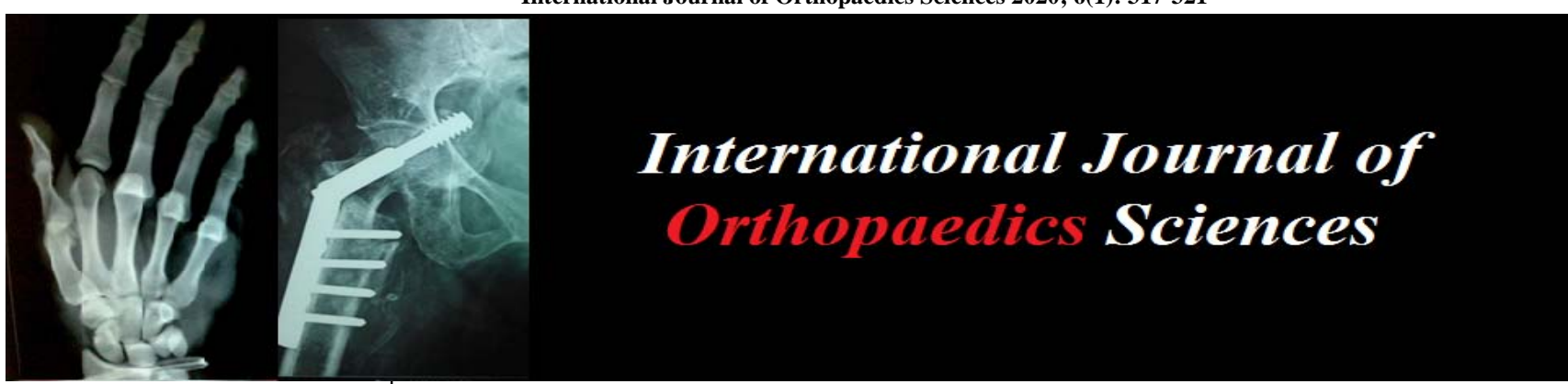

E-ISSN: 2395-1958

P-ISSN: 2706-6630

IJOS 2020; 6(1): 317-321

(C) 2020 IJOS

www.orthopaper.com

Received: 14-11-2019

Accepted: 19-12-2019

Dr Ameet P Kulkarni MBBS, PG D' Ortho, DNB Resident, Department of Orthopedics, Manipal Hospital, Old Airport Road, Bengaluru,

Karnataka.

Dr. Abhishek Mannem MS Ortho Registrar, Department of Orthopedics, Manipal Hospital, old Airport Road, Bengaluru, Karnataka.

Dr Mallinath Gidaganti MS Ortho (AIIMS) Consultant, Department of Orthopedies, Manipal Hospital, old Airport Road, Bengaluru, Karnataka
Corresponding Author: Dr. Abhishek Mannem MS Ortho Registrar, Department of Orthopedics, Manipal Hospital, old Airport Road, Bengaluru, Karnataka, India

\section{A percutaneous repair technique of closed Tendo- Achilles rupture using suture anchors and fiber wire prati technique a retrospective functional outcome study of 20 cases}

\author{
Dr. Ameet P Kulkarni, Dr. Abhishek Mannem and Dr. Mallinath \\ Gidaganti
}

DOI: https://doi.org/10.22271/ortho.2020.v6.i1f.1881

Abstract

Background: Achilles tendon is the strongest and thickest tendon in the human body. Rupture of the Achilles tendon usually occurs in middle-aged population following recreational activities. Incidence rate of these ruptures range from 6 to 18 per 100000 population. Several operative and non-operative treatment options are available. Operative methods include percutaneous, mini-open, open Achilles repair etc. Open repairs carry an increased risk of wound healing problems, whereas minimally invasive techniques are reported to have an increased risk of iatrogenic nerve injury.

Materials and Methods: Twenty patients with acute closed traumatic Tendo Achilles rupture were operated using above mentioned technique between January 2010 to June 2017 in Bangalore out of which 15 were males and 5 were females. Patient was put on anterior below knee slab in plantar flexion of the foot for 2 weeks followed by walking below knee cast for 4 weeks. Patients were followed up at 6 weeks, 3 months, 6 months and 1 year following surgery and complications if any were observed. Postoperative AOFAS ankle - hindfoot score was taken at last follow up.

Result: Percutaneous Tendo Achilles repair has a good outcome in $90 \%$ of the cases. There was one case with complication. The complication was sural nerve hypoesthesia but with no functional disability. The average AOFAS score was 89\% (76-92) in which 65\% (13) were considered excellent, 30\% (6) were considered good and $05 \%$ (1) were considered fair outcome. There were no re-ruptures.

Conclusion: We present our technique of percutaneous Tendo Achilles repair which has minimal wound and nerve injury complications, early return to activities with a good functional outcome, distal bony fixation with the use of suture anchors reducing the number of suture-tendon interfaces which in turn reduces the chance of failure. It also decreases overall operative time. However further studies are required.

Keywords: Tendo Achilles, Closed rupture, Percutaneous repair, Suture anchors, Criss-cross suturing, AOFAS Score (American Orthopaedic foot and ankle society)

\section{Introduction}

Achilles tendon is the strongest and thickest tendon in the human body ${ }^{[1]}$, which takes its name Achilles, from Homer's Iliad. Ruptures of the Achilles tendon are the most common tendon injuries, usually occurring in middle-aged population. Incidence of ruptures range from 6 to 18 per 100000 population ${ }^{[3,4,5]}$. Males are more likely than females to suffer this injury. The male-to-female ratio has been estimated to range from 1.7:1 to 30:1 ${ }^{[6]}$. For acute ruptures, the mean age for men and women was 43.8 years and 55.1 years respectively and there were similar rates of athletic activity as the causative factor in men $(80.5 \%)$ and women $(71.4 \%)^{[7]}$. The injury occurs when a strong dorsiflexion force is applied to the ankle as the triceps surae contracts to plantarflex the ankle, an eccentric contraction. Even though the rupture seemingly occurs as consequence of a traumatic insult on a nevertheless healthy tendon, in reality it may be the end result of a single eccentric contraction on a tendinopathic yet often clinically asymptomatic tendon. Achilles tendon injury is common in the watershed area, approximately 2-6 cm from its insertion on the calcaneal tuberosity. 
Rupture occurs in this watershed area approximately $75 \%$ of the time. Ruptures can also occur at the distal insertion site $(10 \%-20 \%)$ and at the myotendinous junction (5\%-15\%) ${ }^{[8]}$.

The incidence of acute Achilles tendon ruptures is on the rise but there is still controversy regarding the most optimal treatment plan. Debate about nonoperative vs surgical repair for acute ruptures, minimally invasive vs traditional open repair, and early functional rehabilitation protocols instead of a more traditional rehabilitation programme are the arguments that continue to exist in the domain of treatment. Several operative and non-operative treatment options are available. Nonoperative treatment consists of brief immobilization, followed by early rehabilitation and early weight bearing. This line of treatment is reserved for lower demand patients and patients with high surgical risk. Operative methods include percutaneous Achilles repair, mini-open repair, open Achilles repair, hybrid repair and endoscopic repair.

It is important to have a validated patient reported outcome system to complement clinical outcomes. The SF-36 quality of life, the Hannover score, AOFAS Hindfoot score ${ }^{[9]}$, the Foot and Ankle Outcome Score, and the Achilles tendon total rupture score (ATRS) are just a few of the myriad patient outcome scores that have been used to evaluate the function after Achilles tendon rupture repair. AOFAS score has been used in our study to assess the functional outcome after surgery.

The purpose of this study is to describe our new percutaneous technique of surgically managing Achilles tendon rupture using suture anchor and crisscross suturing with fiber wire or ethibond 2-0. We also discussed the comparative outcomes with other techniques, risks and methods to avoid iatrogenic nerve injury.

\section{Indications}

This technique is applied for Achilles tendon ruptures 2-6 cm from the insertion site and bony avulsions of calcaneum in all patients. This technique along with brief period of immobilization and early functional rehabilitation may even be considered for old and sedentary patients, especially in patients with significant medical co-morbidities that may impact wound healing.

\section{Materials and Methods}

Between January 2010 and June 2017, twenty patients with rupture of Achilles tendon in Bangalore were treated using the below mentioned technique. Fifteen patients were male, and five patients were females. Out of the twenty patients two of them were professional athletes. The mean age of the patients was 50.75 years. The mean time between the injury and the index surgery was 3 weeks. All the patients had closed injuries to the Achilles tendon. Patients with old scar marks, previous Achilles tendon injuries treated conservatively or by any surgical methods, sural nerve injury and infection were excluded from this study.

The injuries were confirmed both clinically and radiologically. All the patients showed palpable defect within the Achilles tendon and a positive Thompsons test. All the patients underwent AP and lateral radiographs of the ankle to look for any bony avulsions from calcaneum and USG of the Achilles tendon to confirm the tear.

All the patients in this study were followed at 2 weeks, 4 weeks, 6 weeks, 3 months, 6 months and 1 year following surgery. AOFAS ankle-hind foot score was used for functional evaluation at the last follow-up of 1 year. According to kitoaka et al. ${ }^{[9]}$ results were rated as excellent
(100-90 points), good (89-80), fair $(79-70)$, or poor $(<70)$. Infection, wound breakdown, nerve injury, operating time, weightbearing and Tendon re-ruptures were assessed after repair with this technique.

\section{Technique}

\subsection{Patient positioning:}

Under spinal anesthesia patient is placed in lateral or prone position after applying thigh tourniquet on a standard operative table. All bony prominences were well padded. The operative limb is prepared and draped in a sterile fashion below the knee joint to allow for intraoperative assessment and proper tensioning of the repair. Antibiotic prophylaxis was given before tourniquet inflation. Clinical examination is carried out to precisely locate the defect and is marked with a sterile marker.

\subsection{Operative technique}

The injured limb was positioned in 45 degrees of knee flexion and 15 degrees of plantar flexion at the ankle joint. Either one or Two suture anchors with fiber wire or 2-0 ethibond and an epidural catheter with needle of size $18 \mathrm{G}$ are required to perform this procedure as shown in figure 1.

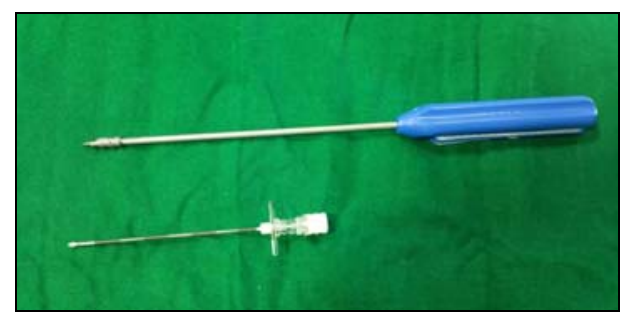

Fig 1: Either one or Two suture anchors with fiber wire and an epidural catheter with needle of size $18 \mathrm{G}$ are required to perform this procedure

A $1 \mathrm{~cm}$ stab incision is made on medial and lateral aspect of tendo Achilles insertion on calcaneal tuberosity were made and the suture anchor is inserted into the calcaneum. Both the free ends of the fiber wire are identified and crisscrossed across the distal tendo Achilles stump using the epidural needle after gently palpating the tendon with thumb and index finger of the opposite hand to make sure that tendon is caught in total and epidural needle is passed through the center of the tendon as sural nerve is anterior and lateral to the Tendo Achilles at this level. The exit point of the fiber wire from the distal stump after crisscrossing will be from the medial and lateral ends of the stump as shown in the figure 2.

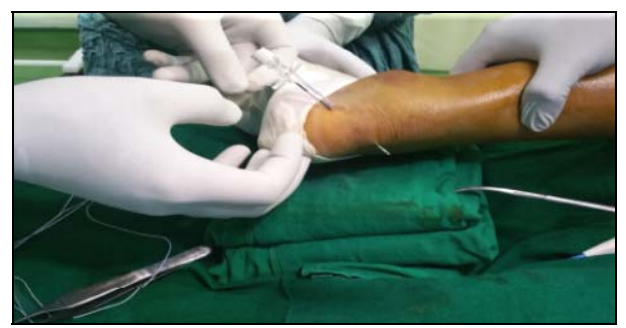

Fig 2: A $1 \mathrm{~cm}$ stab incision is made on medial and lateral aspect of tendo Achilles insertion on calcaneal tuberosity were made and the suture anchor is inserted into the calcaneum. Both the free ends of the fiber wire are identified and crisscrossed across the distal tendo Achilles stump using the epidural needle. The exit point of the fiber wire from the distal stump after crisscrossing will be from the medial and lateral ends of the stump as shown in the figure. 
The Epidural needle again is used to deliver those free ends on to the proximal part crisscrossing at the tear site and exiting through medial and lateral ends of the proximal stump. The ends coming out from the proximal stump are again crisscrossed until the musculotendinous junction $(10-11 \mathrm{~cm}$ from the calcaneal insertion site). At the musculotendinous junction the sural nerve becomes posterior and crosses the lateral border of the tendon, lying in close proximity to the Tendo Achilles. Care should be taken to direct the needle towards anterior and center of the tendon. Above this level sutures were passed through the fascia covering the triceps surae till $15 \mathrm{~cm}$ above its insertion point on the calcaneal tuberosity as shown in figure 3 and figure 4.

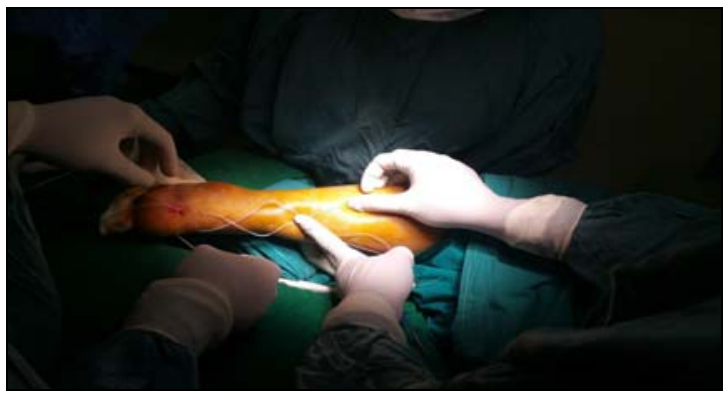

Fig 3: The Epidural needle again is used to deliver those free ends on to the proximal part crisscrossing at the tear site and exiting through medial and lateral ends of the proximal stump in a manner as shown in the figure.

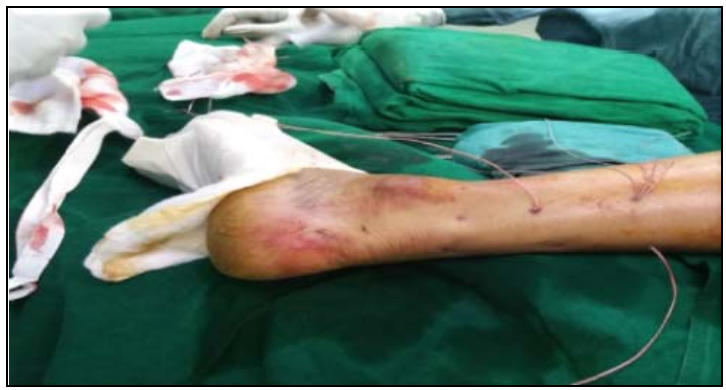

Fig 4: The ends coming out from the proximal stump are again crisscrossed until the musculotendinous junction $(10-11 \mathrm{~cm}$ from the

calcaneal insertion site). Above this level sutures were passed through the fascia covering the triceps surae till $15 \mathrm{~cm}$ above its insertion point on the calcaneal tuberosity as shown in the figure.

The knot was put away from the tear at the musculotendinous junction on either medial or lateral side deep to fascia to avoid puckering as shown in figure 5.

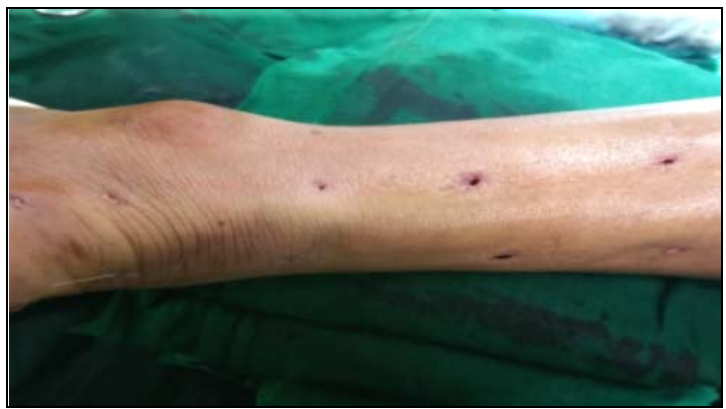

Fig 5: Final picture showing stab incisions for passing epidural catheter with needle and after knotting suture proximally either on the medial or lateral side of tendo Achilles. There is no puckering of skin.
Needling was done at the tear site using epidural needle to enhance bleeding and healing with fibrosis. All Patients were put on anterior below knee slab in plantar flexion of the foot for 2 weeks followed by walking below knee cast for 4 weeks

\section{Result}

Percutaneous Tendo Achilles repair has a good outcome in $90 \%$ of the cases. There was only one case with complication. It was sural nerve hypoesthesia with no functional disability for the patient. The average AOFAS score was 89\% (76-92) in which $65 \%$ (13) were considered excellent, 30\% (6) were considered good and 5\% (01) was considered fair outcome. There were no case of re-ruptures. Functional and cosmetic outcome at 1 year follow up is as showed in Figure 6.

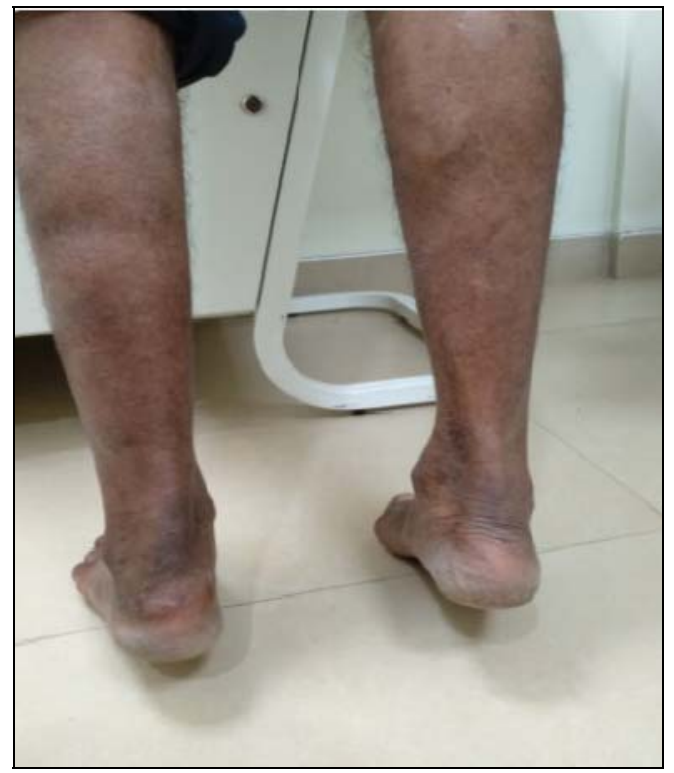

Fig 6: Functional and cosmetic outcome (Left side) at 1 year follow up is as shown in the figure. No scar marks or wound dehiscence with good range of plantar flexion.

\section{Discussion}

Operative repair of Achilles tendon ruptures leads to improved early outcomes, in terms of length, strength, functional activities and reduced tendon elongation compared to non-operative treatment ${ }^{[10,11]}$. It is preferred for treatment in younger, healthier and more active patients. Open repairs carry an increased risk of wound healing problems, whereas minimally invasive techniques are reported to have an increased risk of iatrogenic nerve injury. Percutaneous surgery for Achilles tendon ruptures aims to provide a stable repair, allowing early weight bearing and movement, whilst minimizing the risks of wound breakdown or infection [12, 13, 14].

Ma \& Griffith are the first to describe percutaneous technique of Achilles tendon repair in 1977. This technique developed a reputation for iatrogenic sural nerve injuries, with Klein reporting a $13 \%$ rate of sural nerve involvement, although is still commonly used. The Ma \& Griffith repair consists of a Bunnel suture applied to the proximal tendon and a box suture distally in the stump inserted through 6 para-tendinous stab incisions. They have reported no nerve injuries and good functional outcome ${ }^{[15]}$.

To avoid sural nerve entrapment during percutaneous placement of epidural needle thorough understanding of sural nerve course is required. Extensive literature review has been done to find out safe trajectory of percutaneous needle 
placement. At the level of the ankle, the Achilles tendon ruptures showed greater external rotation than that of nonruptures (15.8 \pm 16.2 degrees vs $5.9 \pm 9.0$ degrees, respectively; $P=.008)$. In a radiological evaluation of Achilles tendon and sural nerve anatomy, Aoife MacMahon et al [16] have found that at the calcaneal insertion, the sural nerve was anterior and lateral to the Tendo Achilles in both cohorts. At 4 $\mathrm{cm}$ proximal to the calcaneal insertion, the sural nerve was anterior and lateral to the AT in both cohorts, but the sural nerve was closer anteriorly $(3.5 \pm 4.0 \mathrm{~mm}$ vs $8.3 \pm 3.1 \mathrm{~mm})$ and farther laterally $(9.0 \pm 2.6 \mathrm{~mm}$ vs $3.4 \pm 3.0 \mathrm{~mm})$ from the AT in ruptures than in no ruptures $(P<.001$ for both comparisons). At the most proximal measurement, the sural nerve was posterior and lateral to the AT in both cohorts, but the sural nerve was farther posteriorly $(3.6 \pm 3.1 \mathrm{~mm}$ vs $1.9 \pm$
$3.4 \mathrm{~mm}, P=.027)$ and laterally $(3.2 \pm 2.7 \mathrm{~mm}$ vs $0.39 \pm 2.4$ $\mathrm{mm}, P<.001)$ from the AT in ruptures than in no ruptures. They therefore concluded that When performing a limitedopen or percutaneous repair, it is important to consider that the ruptured tendon is externally rotated, which can affect both tendon capture and proximity to the sural nerve. They also recommended that external rotation of 11 degrees at the proximal end of the rupture and 16 degrees at the distal end relative to the bimalleolar axis when positioning a percutaneous or limited-open AT repair device. This may be helpful in achieving better tendon capture and minimizing the likelihood of sural nerve injury ${ }^{[16]}$.

Further on comparison with other studies, the results are as shown in the table 1.

Table 1: comparing results of our study with other studies

\begin{tabular}{|c|c|c|c|c|c|c|c|c|c|c|}
\hline \multirow[t]{2}{*}{ Study } & \multicolumn{2}{|c|}{ Wound infection } & \multicolumn{2}{|c|}{ Sural-nerve lesion } & \multicolumn{2}{|c|}{ Operating time in minutes } & \multicolumn{2}{|c|}{ Time-to Mobilize in week } & \multicolumn{2}{|c|}{ Rerupture rate } \\
\hline & Open & Pc /MI & Open & Pc/MI & Open & Pc / MI & Open & Pc / MI & Open & Pc/MI \\
\hline Lim et al ${ }^{[12]}$ & $21 \%$ & nil & nil & $3 \%$ & - & - & - & - & $6 \%$ & $3 \%$ \\
\hline Haji et al ${ }^{[13]}$ & $4.7 \%$ & nil & $1.4 \%$ & $10.5 \%$ & 45.9 & 28.5 & $4-6$ & $2-6$ & $5.7 \%$ & $2.6 \%$ \\
\hline Tejwani et al ${ }^{[14]}$ & $20 \%$ & $3.2 \%$ & nil & $12.9 \%$ & - & - & - & - & - & - \\
\hline Our study & - & nil & - & $5 \%$ & - & 30 & - & 2 & - & nil \\
\hline
\end{tabular}

\section{Conclusion}

There have been many advances in percutaneous and mini open repairs of Tendo Achilles tear to reduce the risk of complications. In the technique described here, distal bony fixation is achieved with the use of suture anchors reducing the number of suture-tendon interfaces which in turn reduces the chance of failure. The study also discusses about the risks and methods to avoid iatrogenic nerve injury and measure the outcome using AOFAS ankle - hindfoot score at 1 year follow up. The percutaneous technique also facilitates decreased overall operative time. This technique allows for patients to recover quickly and return to activities sooner than traditional Achilles repair techniques. Cosmetic outcome is better as compared to other methods. However, further clinical studies are needed

\section{Limitations}

This study does not include pathological and open traumatic causes of Tendo Achilles rupture which may influence AOFAS score postoperatively and the duration from the time of injury to undergoing surgery was not standardised

\section{Recommendation}

This study has sample size of 20 patients with closed traumatic Tendo Achilles rupture. This size may have limited the ability to reach statistical significance in some outcomes. Further study in larger group of patients will be beneficial for recommendations and remedial measures.

Counselling the patients along with explaining the advantages and disadvantages of different management options prior to surgery may be beneficial to both patients and operating surgeon.

\section{References}

1. O'Brien M. Functional anatomy and physiology of tendons. Clin Sports Med. 1992; 11(3):505-520.

2. Ahmad J, Repka M, Raikin SM. Treatment of myotendinous Achilles ruptures. Foot Ankle Int, 2013; 1071100713483115.

3. Huttunen TT, Kannus P, Rolf C, Felländer-Tsai L, Mattila VM. Acute Achilles tendon ruptures: Incidence of injury and surgery in Sweden between 2001 and 2012.

4. Am J Sports Med. 2014; 42:2419-2423.

5. Moller A, Astrom M, Westlin N. Increasing incidence of Achilles tendon rupture. Acta Orthop Scand. 1996; 67:277-279. doi: 10.3109/17453679608996672.

6. Tushar Singhi, Ashith Rao, Abhay Agarwal, Sunil Shetty, Prakash Samant. Modified gift box technique for acute compound posttraumatic Achilles tendon repair in young patients. $\quad$ DOI: http://dx.doi.org/10.18203/issn.24554510.IntJResOrthop20180684

7. Carden D, Noble J, Chalmers J, Lunn P, Ellis J. Rupture of the calcaneal tendon: the early and late management. $\mathrm{J}$ Bone Joint Surg Br. 1987; 69:416-420.

8. Vosseller J, Ellis SJ, Levine DS, Kennedy JG, Elliott AJ, Deland JT et al. Achilles tendon rupture in women. Foot Ankle Int. 2013; 34:49-53.

9. Gwynne-Jones D, Sims M, Handcock D. Epidemiology and outcomes of acute Achilles tendon rupture with operative or nonoperative treatment using an identical functional bracing protocol. Foot Ankle Int. 2011; 32:337-343. doi: 10.3113/FAI.2011.0337.

10. Kitaoka HB, Alexander IJ, Adelaar RS, Nunley JA, Myerson MS, Sanders $\mathrm{M}$ et al. Clinical rating systems for the ankle-hindfoot, midfoot, hallux, and lesser toes. Foot Ankle Int. 1994; 15:349-353. doi: 10.1177/107110079401500701.

11. Cetti R, Christensen SE, Ejsted R, Jensen NM, Jorgensen U. Operative vs nonoperative treatment of Achilles tendon rupture. A prospective randomized study and review of the literature. Am J Sports Med. 1993; 21:791796. doi: $10.1177 / 036354659302100606$

12. Keating JF, Will EM. Operative versus non-operative treatment of acute rupture of tendo Achillis: A Prospective Randomised Evaluation of Functional Outcome. J Bone Joint Surg Br. 2011; 93:1071-1078. doi: 10.1302/0301-620X.93B8.25998.

13. Lim J, Dalal R, Waseem M. Percutaneous vs. open repair of the ruptured Achilles tendon-a prospective randomized controlled study. Foot Ankle Int. 2001; 22:559-568.

14. Haji A, Sahai A, Symes A, Vyas JK. Percutaneous versus open tendo Achilles repair. Foot Ankle Int. 2004; 25:215- 
15. Tejwani NC, Lee J, Weatherall J, Sherman O. Acute achilles tendon ruptures: a comparison of minimally invasive and open approach repairs followed by early rehabilitation. Am J Orthop (Belle Mead NJ). 2014; 43(10):E221-5.

16. Ma GW, Griffith TG. Percutaneous repair of acute closed ruptured achilles tendon: a new technique. Clin Orthop Relat Res. 1977, 247-255.

17. MacMahon A, Deland JT, Do H, Soukup DS, Sofka CM, Demetracopoulos CA et al. MRI Evaluation of Achilles Tendon Rotation and Sural Nerve Anatomy: Implications for Percutaneous and Limited-Open Achilles Tendon Repair. Foot Ankle Int. 2016; 37(6):636-43. Doi: 10.1177/1071100716628915. 